\title{
Chordopoxvirus protein F12 implicated in enveloped virion morphogenesis is an inactivated DNA polymerase
}

\author{
Natalya Yutin ${ }^{1}$, Guilhem Faure ${ }^{1}$ Eugene V Koonin ${ }^{1 *}$ and Arcady R Mushegian²
}

\begin{abstract}
Through the course of their evolution, viruses with large genomes have acquired numerous host genes, most of which perform function in virus reproduction in a manner that is related to their original activities in the cells, but some are exapted for new roles. Here we report the unexpected finding that protein F12, which is conserved among the chordopoxviruses and is implicated in the morphogenesis of enveloped intracellular virions, is a derived DNA polymerase, possibly of bacteriophage origin, in which the polymerase domain and probably the exonuclease domain have been inactivated. Thus, F12 appears to present a rare example of a drastic, exaptive functional change in virus evolution.
\end{abstract}

Reviewers: This article was reviewed by Frank Eisenhaber and Juergen Brosius. For complete reviews, go the Reviewers' Reports section.

Keywords: DNA polymerase, Exaptation, Poxviruses, Evolution of viruses

\section{Findings}

Genomes of large viruses, in addition to a small core of viral hallmark genes, encompass numerous genes that apparently have been acquired from the hosts at different stages of evolution [1-3]. Some of these genes, such as diverse metabolic, repair and signaling enzymes, retain their original biochemical activities that are utilized for virus reproduction. For other gene products, the original function is mechanistically exploited but part of the functionality has been lost during virus evolution converting the gene products into inhibitors or modulators of the respective host pathways, such as programmed cell death or various forms of immunity, a phenomenon often called molecular mimicry and especially well characterized in poxviruses [4-7]. However, several cases have been reported where the acquired host gene seems to have been exapted [8] for a function in virus reproduction that was not obviously related to the original one. For example, the poxvirus D4 protein, a uracil DNA glycosylase,

\footnotetext{
*Correspondence: koonin@ncbi.nlm.nih.gov

'National Center for Biotechnology Information, National Library of Medicine, National Institutes of Health, Bethesda, MD 20894, USA

Full list of author information is available at the end of the article
}

functions as a processivity subunit of the viral DNA polymerase, a role for which the enzymatic activity of D4 is not required [9]. Another case in point is the poxvirus F16 protein which appears to be an inactivated serine recombinase and unexpectedly localizes to the nucleoli of the infected cells although its role in virus reproduction remains obscure [10]. We report here that poxvirus protein $\mathrm{F} 12$ that has been implicated in intracellular enveloped virus (IEV) morphogenesis, and in particular IEV movement along microtubules [11-13], is a derived DNA polymerase in which both the polymerase and the exonuclease activities apparently were abrogated as a result of mutational replacement of catalytic amino acid residues. This finding reveals another, striking case of exaptation in virus evolution.

\section{Chordopoxvirus protein F12 is an inactivated homolog of Family B DNA polymerases \\ In the course of a survey of the evolutionary provenance of poxvirus proteins, we unexpectedly observed that PSI-BLAST searches against the non-redundant data- base (NCBI, NIH, Bethesda) initiated with the amino acid sequence of Vaccinia virus (VACV) protein F12}


(GenBank Accession No Q80HX6) detected, in addition to the highly significant similarity to the homologs from all chordopoxviruses, a marginal, not statistically significant similarity to several identified or putative DNAdependent DNA polymerases (DNAPs) from plant and fungal mitochondrial plasmids and bacteriophages. To further investigate the possible homology of F12 and DNAPs, we used the sequence of the F12 homolog encoded by the most distant from VACV, early branching chordopoxvirus, the Nile Crocodile Virus (NCV) (YP_784228), as the query for a new PSI-BLAST search. This third iteration of this search identified statistically significant similarity (E-value $<0.001$ ) between F12 and a variety of organellar plasmid and phage DNAPs. Further sequence analysis was performed using the HHPred method which compares Hidden Markov Model profiles derived from the multiple alignment of readily detectable homologs of the query protein to databases of profiles of structurally characterized protein families. The HHPred search initiated with the sequence of either VACV F12 or the NCV homolog of F12 consistently yielded alignments with the Bacillus subtilis phage Phi29 DNAP (pdb 2py5), with a probability values greater than 98.5 , which is considered strong evidence of homology, and a close correspondence between secondary structure elements (see Additional file 1). Somewhat weaker similarity was observed with a variety of plasmid-encoded DNAPs.
Similar results were obtained with the Phyre2 method for protein structure prediction (see Additional file 2).

Taken together, these observations indicate that chordopoxvirus F12 proteins are homologs of family B DNAPs, with the strongest sequence similarity observed with the protein-primed DNAPs of phages and organellar plasmids. The family B DNAPs consist of an N-terminal 3'-5'-exonuclease (Exo) domain and the $\mathrm{C}$-terminal polymerase moiety that encompasses the Palm, Fingers and Thumb domains $[14,15]$. The Exo and Palm domains show high level of sequence conservation throughout the family whereas the Fingers and Thumb domains are poorly conserved. Examination of the multiple alignment of the F12 proteins with the DNAPs shows that most of the amino acid residues that belong to the conserved motifs of the Palm domain and contribute to catalysis are replaced in F12 indicating that the polymerase activity has been lost in the viral proteins (Figure 1). The catalytic motifs of the Exo domain show a greater degree of conservation in F12, so the possibility that some level of exonuclease activity persists in some of the viral proteins cannot be ruled out (Figure 1).

Poxviruses encode their own, functional family B DNAPs that is essential for virus replication [6]. The PFAM families encompassing the Exo and Palm domains of these enzymes were also observed in HHPred searches but the level of similarity between

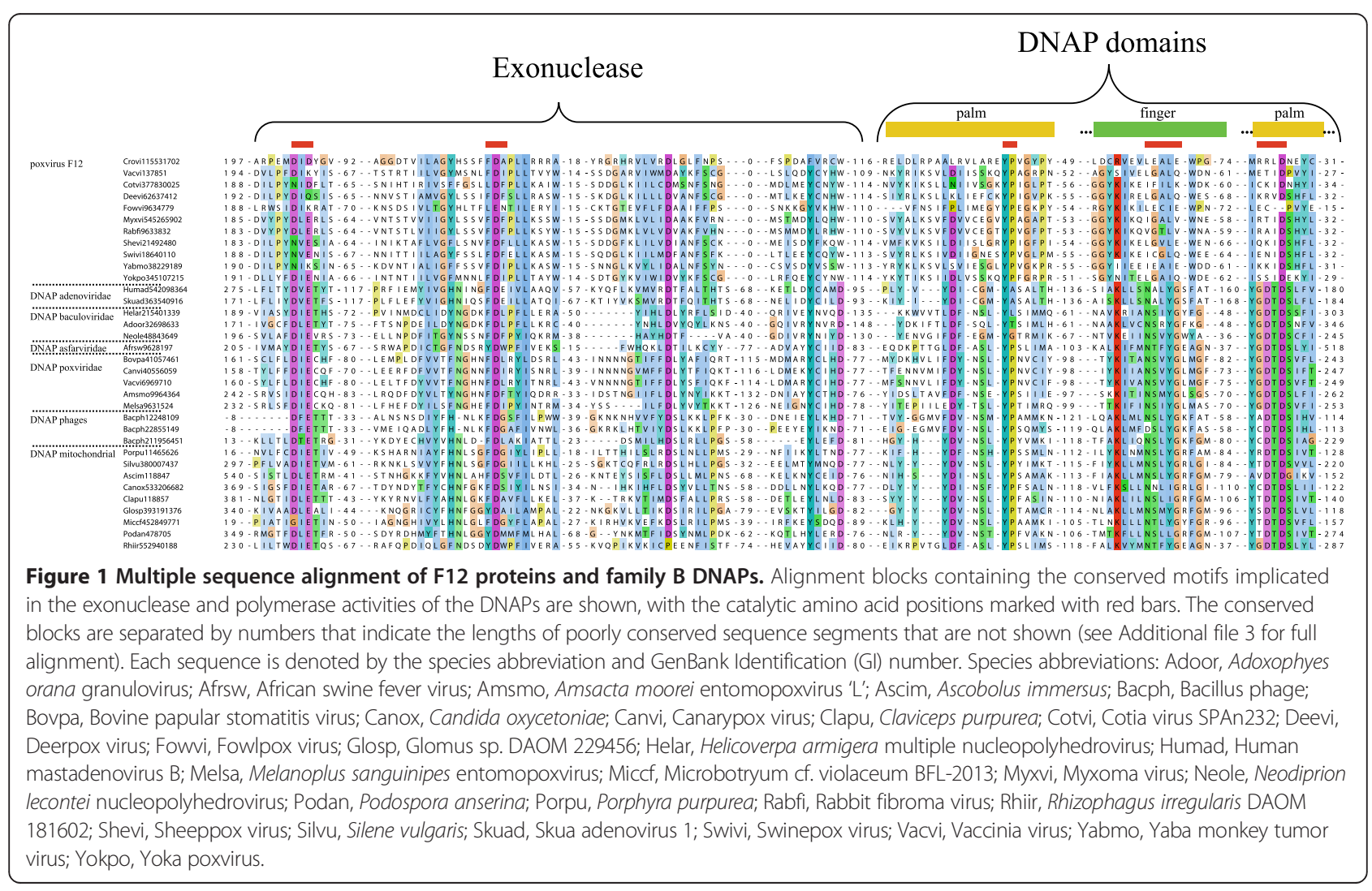


F12 proteins and virus DNAPs was substantially lower than that between F12 and phage DNAPs, indicating that F12 is unlikely to have arisen via a within-genome duplication of poxvirus DNAPs.

The multiple alignment of various DNAPs and chordopoxvirus F12 proteins (see Additional file 3) was used to infer a phylogenetic tree in which F12 clustered with the protein-primed phage and plasmid DNAPs, albeit with a moderate bootstrap support (Figure 2). This phylogeny should be interpreted with caution, especially given the acceleration of evolution of the F12 gene, likely associated with the inactivation of the enzymatic domains. Nevertheless, together with the results of sequence and structure similarity searches, these findings suggest the possibility that a bacteriophage DNAP gene was acquired by the ancestral chordopoxvirus via horizontal gene transfer. This acquisition was then followed by exaptation for a role in IEV morphogenesis and transport along microtubules [11] and the concomitant disruption of the DNAP catalytic centers. A notable parallel is the likely acquisition of the F16 gene, located in the same region of chordopoxvirus genomes, from a bacteriophage gene, followed by the elimination of the enzymatic (recombinase) activity, also most likely early in poxvirus evolution [10]. At least one other gene that is conserved among chordopoxviruses, G6, apparently was acquired from a bacterial source [16]. Thus, the origin of chordopoxviruses seems to have involved a substantial contribution from bacteria and their viruses.

\section{No relationship between F12 and the TPR repeats of kinesin light chains}

The poxvirus F12 protein has been claimed to share functionally relevant similarity with the tetratricopeptide repeats (TPR) region of kinesin light chains (KLC) although no quantitative evidence has been presented in

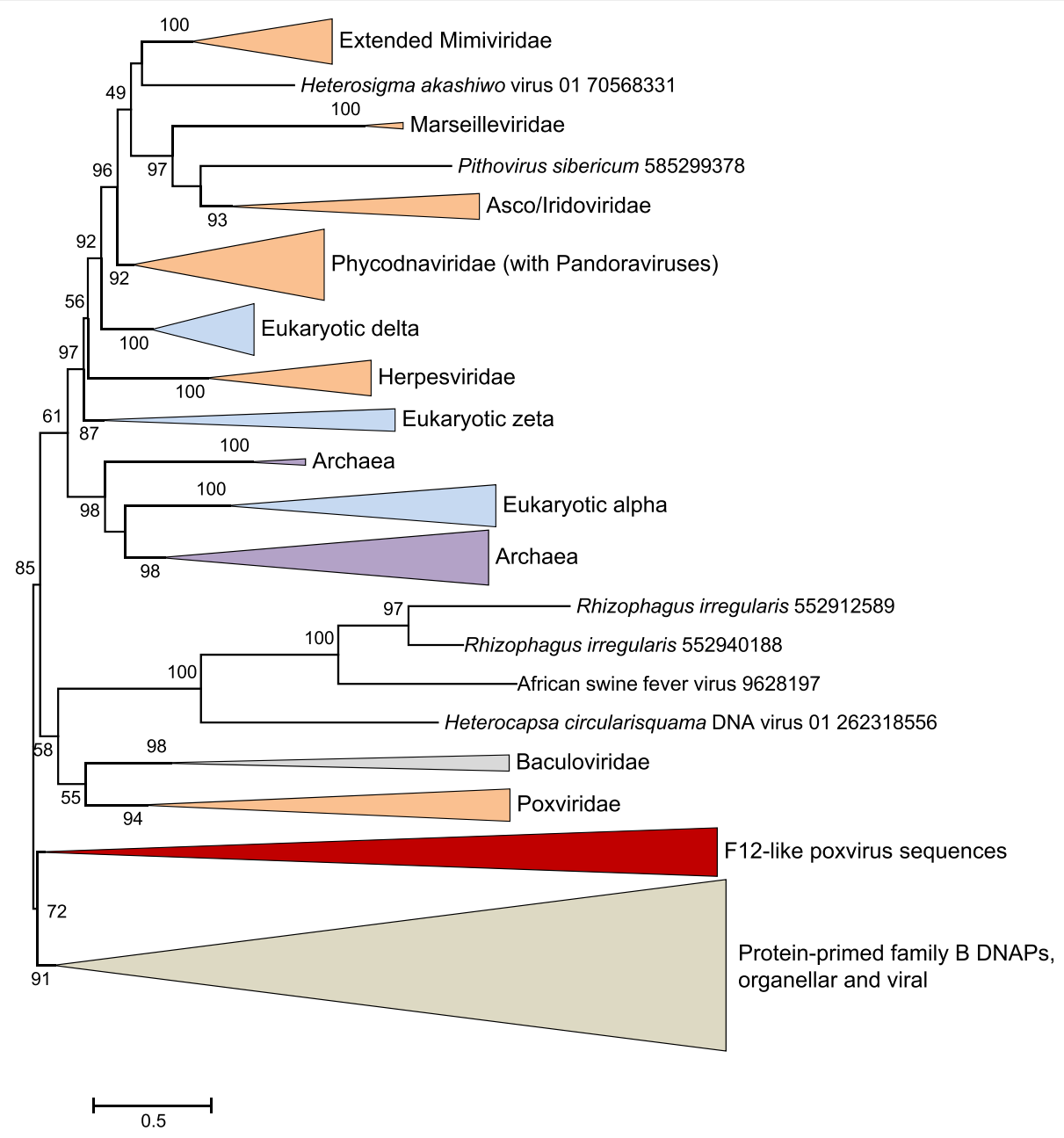

Figure 2 Phylogenetic tree of the family B DNAPs including F12 proteins. For the multiple alignment used for the phylogenetic analysis, see Additional file 3. Multiple sequences from several clades are collapsed and shown with triangles. Approximate bootstrap values calculated by FastTree are shown for each internal branch. 
support of this connection [13]. However, no similarity to TPR repeats was detected in our search of the Conserved Domain Database at the NCBI or using the more sensitive HHPred search. More important, the presence of all-alpha TPR repeats is incompatible with the homology of F12 with the alpha-beta DNAP domains or the predicted secondary structure of F12 (Additional file 1). Identification of multiple TPRs has been reported also for two other chordopoxvirus proteins that contribute to IEV maturation and motility, namely for E2, which forms a complex with F12 [11], and for A36 [13]. Using a TPR predictor tool, we detected no TPRs in F12, E2, and A36 whereas multiple TPRs were confidently predicted in KLC (Additional file 4).

It has been reported that F12 is required for the recruitment of kinesin-1 which enables the movement of IEV along microtubules in VACV-infected cells and that deletion of the purported TPRs in F12 abrogated kinesin binding [13]. The findings described here do not conflict with these experimental observations but suggest that the interaction between F12 and kinesin is mediated by the derived DNAP domains that are unrelated to TPRs.

\section{Conclusions}

We report here the unexpected finding that a chordopoxvirus protein implicated in IEV morphogenesis and intracellular motility is a derived, inactivated DNAP. The results of sequence comparison and phylogenetic analysis suggest an evolutionary scenario in which the F12 gene evolved from an acquired bacteriophage DNAP gene, with both exonuclease and polymerase activities apparently abrogated as indicated by the disruption of the respective catalytic sites. Alternative routes of evolution, such as duplication and subsequent inactivation of the ancestral poxvirus DNAP, cannot be formally ruled out but appear much less likely. Inactivated DNAPs has been described previously in archaea and eukaryotes $[15,17,18]$, and recently, it has been shown that the UL8 subunit of herpes virus DNA primase is an inactivated family B DNAP [19]. However, in all these cases, the inactivated polymerases still function in DNA replication or repair, conceivably interacting with some of the same partners as active DNAPs do. The poxvirus F12 seems to present the case where an inactivated DNAP is exapted for a completely new role in a different cellular location. Characterization of potential structural features linking the complexes of F12 with its protein partners and possibly with replication complexes could be an intriguing experimental task.

\section{Methods}

Sequence analysis and phylogenetic tree construction

The non-redundant database of protein sequences at the NCBI was searched using the PSI-BLAST program [20].
Protein sequences were aligned using MUSCLE [21]; gapped columns (more than $30 \%$ of gaps) and columns with low information content were removed from the alignment [22]. For the purpose of visualization, alignment columns were colored using Jalview [23], with the ClustalX coloring conventions [24] and conservation color increment set at 10. Profile-against-profile searches were performed using the HHPred method [25]. Protein structure prediction was performed using the Phyre2 software [26]. Phylogenetic analysis was performed using the FastTree program with default parameters (JTT evolutionary model, discrete gamma model with 20 rate categories) [27]. The TPRs were predicted using the TPRpred software [28].

\section{Reviewers' comments}

\section{Reviewer 1: Frank Eisenhaber, Bioinformatics Institute,} Singapore

The proposed MS describes the discovery of chordopoxvirus F12 phylogenetic history as a inactivated DNA polymerase. The MS is well written, logically rigorous and worth being published.

Authors' response: We appreciate this laconic yet encouraging review.

\section{Reviewer 2: Juergen Brosius, University of Muenster}

The finding that chordopoxvirus protein F12 is an acquisition of a DNA polymerase of likely bacterial origin and a subsequent exaptation as a protein with different viral function(s) is very interesting.

Unlike other examples of DNA polymerases (DNAPs) that lost their central function but still are involved in DNA replication or repair, the F12 protein has been recruited (fortuitously) into an entirely different viral task, namely intracellular virus morphogenesis and transport. As an aside, the term inactivated DNAPs is somewhat imprecise and misleading as they still exhibit a function. Perhaps, partially inactivated would be a better term.

Authors' response: The term "inactivated" pertains to enzymatic activities not to the loss of any functionality. We thought this should be clear but in order to make the text unequivocal in this regard, we modified the penultimate sentence of the introductory paragraph as follows:

"We report here that poxvirus protein F12 that has been implicated in intracellular enveloped virus (IEV) morphogenesis, and in particular IEV movement along microtubules [11-13], is a derived DNA polymerase in which both the polymerase and the exonuclease activities apparently were abrogated as a result of mutational replacement of catalytic amino acid residues." Language to the same effect was also inserted into the Conclusions. 
It is not clear whether any of the bona fide family B DNAPs of chordopoxviruses are shown in any of the alignments, although it is stated that there is most likely no relationship to invoke a viral gene duplication event.

Authors' response: The alignment in Figure 1 actually includes 5 poxvirus DNAP sequences that are explicitly marked as such. The problem probably is with the truncation of the figure in the original pdf file. We are taking care of this in the revision.

The term horizontal transfer is missing from the text. Does it mean that acquisition of an originally bacteriophage DNAP gene by a virus infecting eukaryotic cells cannot be considered HGT?

Authors' response: We agree that horizontal gene transfer is an appropriate term to describe this case and include it in the revised text.

In the pdf version of the manuscript, Figure 1 is truncated. Among other things, the important subdivisions on the left margins can only be guessed.

Authors' response: We regret the inconvenience caused by poor formatting of the figure. We include a version with wider margins which should eliminate the problem.

\section{Additional files}

Additional file 1: The HHPred output for the F12 homolog of CNV. Additional file 2: Phyre 2 results for VACV F12.

Additional file 3: Multiple alignment used for the construction of the phylogenetic tree in Figure 2.

Additional file 4: TPRpred results for the relevant poxvirus proteins and kinesin light chains.

\section{Abbreviations}

DNAP: DNA polymerase; Exo: Exonuclease; IEV: Intracellular enveloped virus; KLC: Kinesin light chain; NCV: Nile crocodile virus; TPR: TetratricoPeptide repeat; VACV: Vaccinia virus.

\section{Competing interests}

The authors declare that they have no competing interests.

\section{Authors' contributions}

ARM made the original observation and incepted the study; NY, GF and EVK performed data analysis; EVK wrote the manuscript that was read and approved by all authors. All authors read and approved the final manuscript.

\section{Authors' information}

Arcady R Mushegian: The views expressed in this article are those of the author in his personal capacity and do not necessarily represent the view of the National Science Foundation or the Government of the United States.

\section{Acknowledgments}

NY, GF and EVK are supported by intramural funds of the US Department of Health and Human Services (to the National Library of Medicine). ARM was supported by the Independent Research/Development program at the National Science Foundation while working on this project.

\section{Author details}

${ }^{1}$ National Center for Biotechnology Information, National Library of Medicine, National Institutes of Health, Bethesda, MD 20894, USA. ${ }^{2}$ Molecular and Cellular Biosciences Division, National Science Foundation, Arlington, VA 22230, USA.
Received: 19 August 2014 Accepted: 17 October 2014

Published: 6 November 2014

\section{References}

1. Koonin EV, Senkevich TG, Dolja W: The ancient Virus World and evolution of cells. Biol Direct 2006, 1:29.

2. Yutin $\mathrm{N}$, Wolf $\mathrm{Yl}$, Koonin EV: Origin of giant viruses from smaller DNA viruses not from a fourth domain of cellular life. Virology 2014, 466-467:38-52.

3. Filee J: Route of NCLDV evolution: the genomic accordion. Curr Opin Virol 2013, 3(5):595-599

4. Senkevich TG, Koonin EV, Bugert JJ, Darai G, Moss B: The genome of molluscum contagiosum virus: analysis and comparison with other poxviruses. Virology 1997, 233(1):19-42.

5. Moss B, Shisler JL, Xiang Y, Senkevich TG: Immune-defense molecules of molluscum contagiosum virus, a human poxvirus. Trends Microbiol 2000, 8(10):473-477.

6. Moss B: Poxviridae: The Viruses and their Replication. In Fields Virology, vol. 2. 4th edition. Edited by Fields BN, Knipe DM, Howley PM, Griffin DE. Philadelphia: Lippincott, Williams \& Wilkins; 2001:2849-2884.

7. Smith GL, Benfield CT, Maluquer de Motes C, Mazzon M, Ember SW, Ferguson BJ, Sumner RP: Vaccinia virus immune evasion: mechanisms, virulence and immunogenicity. J Gen Virol 2013, 94(Pt 11):2367-2392.

8. Gould SJ: The exaptive excellence of spandrels as a term and prototype. Proc Natl Acad Sci U S A 1997, 94(20):10750-10755.

9. Stanitsa ES, Arps L, Traktman P: Vaccinia virus uracil DNA glycosylase interacts with the A20 protein to form a heterodimeric processivity factor for the viral DNA polymerase. J Biol Chem 2006, 281(6):3439-3451.

10. Senkevich TG, Koonin EV, Moss B: Vaccinia virus F16 protein, a predicted catalytically inactive member of the prokaryotic serine recombinase superfamily, is targeted to nucleoli. Virology 2011, 417(2):334-342.

11. Dodding MP, Newsome TP, Collinson LM, Edwards C, Way M: An E2-F12 complex is required for intracellular enveloped virus morphogenesis during vaccinia infection. Cell Microbiol 2009, 11(5):808-824.

12. Johnston SC, Ward BM: Vaccinia virus protein F12 associates with intracellular enveloped virions through an interaction with A36. J Virol 2009, 83(4):1708-1717.

13. Morgan GW, Hollinshead M, Ferguson BJ, Murphy BJ, Carpentier DC Smith GL: Vaccinia protein F12 has structural similarity to kinesin light chain and contains a motor binding motif required for virion export. PLoS Pathog 2010, 6(2):e1000785.

14. Braithwaite DK, Ito J: Compilation, alignment, and phylogenetic relationships of DNA polymerases. Nucleic Acids Res 1993, 21(4):787-802.

15. Tahirov TH, Makarova KS, Rogozin IB, Pavlov YI, Koonin EV: Evolution of DNA polymerases: an inactivated polymerase-exonuclease module in Pol epsilon and a chimeric origin of eukaryotic polymerases from two classes of archaeal ancestors. Biol Direct 2009, 4:11.

16. Senkevich TG, Wyatt LS, Weisberg AS, Koonin EV, Moss B: A conserved poxvirus NlpC/P60 superfamily protein contributes to vaccinia virus virulence in mice but not to replication in cell culture. Virology 2008, 374(2):506-514.

17. Rogozin IB, Makarova KS, Pavlov YI, Koonin EV: A highly conserved family of inactivated archaeal B family DNA polymerases. Biol Direct 2008, 3:32.

18. Makarova KS, Krupovic M, Koonin EV: Evolution of replicative DNA polymerases in archaea and their contributions to the eukaryotic replication machinery. Frontiers Microbiol 2014, doi:10.3389/fmicb.2014.00354.

19. Kazlauskas D, Venclovas C: Herpesviral helicase-primase subunit UL8 is inactivated B-family polymerase. Bioinformatics 2014, 30(15):2093-2097.

20. Altschul SF, Madden TL, Schaffer AA, Zhang J, Zhang Z, Miller W, Lipman DJ: Gapped BLAST and PSI-BLAST: a new generation of protein database search programs. Nucleic Acids Res 1997, 25(17):3389-3402.

21. Edgar RC: MUSCLE: multiple sequence alignment with high accuracy and high throughput. Nucleic Acids Res 2004, 32(5):1792-1797.

22. Yutin N, Makarova KS, Mekhedov SL, Wolf YI, Koonin EV: The deep archaeal roots of eukaryotes. Mol Biol Evol 2008, 25(8):1619-1630.

23. Waterhouse AM, Procter JB, Martin DM, Clamp M, Barton GJ: Jalview Version 2-a multiple sequence alignment editor and analysis workbench. Bioinformatics 2009, 25(9):1189-1191. 
24. Thompson JD, Gibson TJ, Higgins DG: Multiple sequence alignment using ClustalW and ClustalX. Curr Protoc Bioinformatics 2002, Chapter 2:Unit 23.

25. Soding J: Protein homology detection by HMM-HMM comparison. Bioinformatics 2005, 21(7):951-960.

26. Kelley LA, Sternberg MJ: Protein structure prediction on the Web: a case study using the Phyre server. Nat Protoc 2009, 4(3):363-371.

27. Price MN, Dehal PS, Arkin AP: FastTree 2-approximately maximum-likelihood trees for large alignments. PLOS ONE 2010, 5(3):e9490.

28. Karpenahalli MR, Lupas AN, Soding J: TPRpred: a tool for prediction of TPR-, PPR- and SEL1-like repeats from protein sequences. BMC Bioinformatics 2007, 8:2.

doi:10.1186/1745-6150-9-22

Cite this article as: Yutin et al:: Chordopoxvirus protein F12 implicated in enveloped virion morphogenesis is an inactivated DNA polymerase. Biology Direct 2014 9:22.

\section{Submit your next manuscript to BioMed Central and take full advantage of:}

- Convenient online submission

- Thorough peer review

- No space constraints or color figure charges

- Immediate publication on acceptance

- Inclusion in PubMed, CAS, Scopus and Google Scholar

- Research which is freely available for redistribution 